\title{
DE Canum Venaticorum: a bright, eclipsing red dwarf-white dwarf binary
}

E. J. M. van den Besselaar ${ }^{1}$, R. Greimel ${ }^{2}$, L. Morales-Rueda ${ }^{1}$, G. Nelemans ${ }^{1}$, J. R. Thorstensen ${ }^{3}$, T. R. Marsh ${ }^{4}$, V. S. Dhillon ${ }^{5}$, R. M. Robb ${ }^{6}$, D. D. Balam ${ }^{6}$, E. W. Guenther ${ }^{7}$, J. Kemp ${ }^{8}$, T. Augusteijn ${ }^{9}$, and P. J. Groot ${ }^{1}$

1 Department of Astrophysics, IMAPP, Radboud University Nijmegen, PO Box 9010, 6500 GL Nijmegen, The Netherlands e-mail: [besselaar;lmr;nelemans;pgroot] @astro.ru.nl

2 Isaac Newton Group of Telescopes, Apartado de correos 321, 38700 Santa Cruz de la Palma, Spain e-mail: greimel@ing.iac.es

3 Department of Physics and Astronomy, Dartmouth College, 6127 Wilder Laboratory Hanover, NH 03755, USA e-mail: thorsten@partita.dartmouth.edu

4 Department of Physics, University of Warwick, Coventry CV4 7AL, UK e-mail: t.r.marsh@warwick.ac.uk

5 Department of Physics and Astronomy, University of Sheffield, Sheffield S3 7RH, UK e-mail: vik.dhillon@sheffield.ac.uk

6 Department of Physics and Astronomy, University of Victoria, Victoria, BC, V8W 3P6, Canada e-mail: robb@uvic.ca; cosmos@uvvm.uvic.ca

7 Thüringer Landessternwarte Tautenburg, Sternwarte 5, 07778 Tautenburg, Germany e-mail: guenther@tls-tautenburg.de

8 Joint Astronomy Centre 660 N. A’ohoku Place University Park Hilo, Hawaii 96720, USA e-mail: j . kemp@jach.hawaii.edu

9 Nordic Optical Telescope, Apartado 474, 38700 Santa Cruz de La Palma, Spain e-mail: tau@not.iac.es

Received 14 August 2006 / Accepted 16 January 2007

\begin{abstract}
Context. Close white dwarf-red dwarf binaries must have gone through a common-envelope phase during their evolution. DE CVn is a detached white dwarf-red dwarf binary with a relatively short $(\sim 8.7 \mathrm{~h})$ orbital period. Its brightness and the presence of eclipses makes this system ideal for a more detailed study.

Aims. From a study of photometric ${ }^{\star}$ and spectroscopic observations of DE CVn we derive the system parameters that we discuss in the framework of common-envelope evolution.

Methods. Photometric observations of the eclipses are used to determine an accurate ephemeris. From a model fit to an average lowresolution spectrum of DE CVn, we constrain the temperature of the white dwarf and the spectral type of the red dwarf. The eclipse light curve is analysed and combined with the radial velocity curve of the red dwarf determined from time-resolved spectroscopy to derive constraints on the inclination and the masses of the components in the system.

Results. The derived ephemeris is $\mathrm{HJD}_{\min }=2452784.5533(1)+0.3641394(2) \times E$. The red dwarf in DE CVn has a spectral type of M3V and the white dwarf has an effective temperature of $8000 \mathrm{~K}$. The inclination of the system is $86_{-2}^{+30}$ and the mass and radius of the red dwarf are $0.41 \pm 0.06 M_{\odot}$ and $0.37_{-0.007}^{+0.06} R_{\odot}$, respectively, and the mass and radius of the white dwarf are $0.51_{-0.02}^{+0.06} M_{\odot}$ and $0.0136_{-0.0002}^{+0.0008} R_{\odot}$, respectively.

Conclusions. We found that the white dwarf has a hydrogen-rich atmosphere (DA-type). Given that DE CVn has experienced a common-envelope phase, we can reconstruct its evolution and we find that the progenitor of the white dwarf was a relatively lowmass $\operatorname{star}\left(M \leq 1.6 M_{\odot}\right)$. The current age of this system is $3.3-7.3 \times 10^{9}$ years, while it will take longer than the Hubble time for DE CVn to evolve into a semi-detached system.
\end{abstract}

Key words. stars: individual: DE CVn - stars: binaries: eclipsing - stars: binaries: close - stars: late-type - stars: white dwarfs stars: fundamental parameters

\section{Introduction}

Large gaps remain in our knowledge of binary stellar evolution that affect our understanding of not only evolved compact binaries, but also of phenomena such as supernovae type Ia

* The photometric observations are only available in electronic form at the CDS via anonymous ftp to

cdsarc.u-strasbg.fr $(130.79 .128 .5)$ or via

http://cdsweb.u-strasbg.fr/cgi-bin/qcat?J/A+A/466/1031 explosions, the rate of neutron star-neutron star mergers, and the number of gravitational wave sources in our Galaxy. The poorly understood physics of the common-envelope (CE) phase results in considerable uncertainty in the binary evolution (Paczyński 1976). During the evolution of a binary, the more massive star turns into a giant. When the initial orbital period is small enough ( $\lesssim 10$ years, Taam \& Sandquist 2000), the envelope of the giant will encompass the secondary star. The secondary and the core of the giant will spiral in towards each other in a CE. When 
the envelope is expelled a close binary consisting of the core of the giant, which will evolve towards a white dwarf, and the unevolved secondary star may emerge (see, e.g., Nelemans \& Tout 2005).

The CE phase is expected to be very short $(\lesssim 1000$ years, Taam \& Sandquist 2000) and is therefore virtually impossible to observe directly. To study the effects of this phase it is best to focus on objects that have most probably undergone a CE phase in their past. These we identify with binary systems containing at least one stellar remnant where the current orbital separation is smaller than the radius of the giant progenitor (usually with orbital periods $\leq 1$ day).

Eclipsing close binaries offer the greatest possibility of deriving precise physical parameters of the stars. The masses, radii, and orbital separations give insight into the binary evolution and specifically tell us if a CE phase has happened sometime in their past. Some examples of detached, close white dwarflow-mass main-sequence star (red dwarf) eclipsing binaries are: RR Cae (Bruch 1999), NN Ser (Haefner 1989), EC13471-1258 (O'Donoghue et al. 2003), GK Vir (Green et al. 1986), and RX J2130.6+4710 (Maxted et al. 2004). For a review on detached white dwarf-red dwarf binaries see, e.g., Marsh (2000) and Schreiber \& Gänsicke (2003). The latest list of white dwarfred dwarf binaries is in Morales-Rueda et al. (2005, with ten new systems compared to Marsh 2000). It is necessary to study as many of these systems as possible to be able to compare their characteristics with population synthesis models and to find their space densities as a function of composition (e.g., white dwarf temperature, spectral type, age).

DE CVn (RX J1326.9+4532) is a relatively unstudied, bright $(V=12.8)$ eclipsing binary. It was first discovered as an X-ray source by ROSAT (Voges et al. 1999) and has a proper motion of $-0.198 \pm 0.002^{\prime \prime} \mathrm{yr}^{-1}$ in right ascension and $-0.178 \pm 0.003^{\prime \prime} \mathrm{yr}^{-1}$ in declination, as given in the USNO-B1 catalog (Monet et al. 2003). This object was first studied photometrically by Robb \& Greimel (1997). From the light curve and the unequal maxima they derived an orbital period of 0.364 days. The asymmetry in their light curve needed a star spot to accurately model the light curve. Robb \& Greimel (1997) measured eclipse depths of $0.054 \pm 0.010$ magnitude in the $R$-band and $0.128 \pm 0.029$ magnitude in the $V$-band.

Holmes \& Samus (2001) obtained UBVRI photometry for five nights in June 2000. They confirm the dependence of the eclipse depth with colours and found minimum depths of the eclipse of $0.10 \mathrm{mag}$ in $I, 0.15$ in $R, 0.30$ in $V, 0.60$ in $B$, and 1.00 in $U$. The differences with wavelength band indicate that the two stars have very different colours.

We note a difference in the eclipse depths as quoted by Holmes \& Samus (2001) compared to the values from Robb \& Greimel (1997). Although Holmes \& Samus (2001) give their values as being eclipse depths, when looking at the light curve we suggest that they have taken the difference between minimum and maximum light instead of the difference between the start and minimum of the eclipse, which is used by Robb \& Greimel (1997) and in the present work.

DE CVn consists of an M-type star with a spectroscopically unseen companion, presumably a white dwarf. Throughout this paper we will refer to the $\mathrm{M}$ dwarf as the secondary component and the probable white dwarf as the primary component. In Sect. 2 we describe our observations and reductions. The results are shown in Sect. 3, and the conclusions are given in Sect. 4.
Table 1. Log of the photometric data of DE CVn.

\begin{tabular}{lllrr}
\hline \hline $\begin{array}{l}\text { Date } \\
\text { (1) }\end{array}$ & Tel & Filter & $T(\mathrm{~s})$ & $\#$ \\
\hline 12 Apr. 1997 & UVic & $R$ & $(4)$ & $(5)$ \\
21 Apr. 1997 & UVic & $R$ & 99 & 173 \\
22 Apr. 1997 & UVic & $R$ & 120 & 168 \\
24 Apr. 1997 & UVic & $R$ & 140 & 176 \\
2 May 1997 & UVic & $R$ & 99 & 175 \\
8 May 1997 & UVic & $R$ & 99 & 37 \\
8 May 1997 & UVic & $V$ & 120 & 39 \\
9 May 1997 & UVic & clear & 34 & 164 \\
10 May 1997 & UVic & clear & 33 & 476 \\
1 Jul. 1998 & UVic & $R$ & 140 & 102 \\
17 Mar. 2000 & UVic & clear & 99 & 52 \\
21 May 2001 & UVic & $R$ & 120 & 160 \\
26 May 2001 & DAO & $B$ & 40 & 110 \\
2 Jun. 2001 & UVic & clear & 40 & 130 \\
21 Jan. 2002 & MDM & $B$ & 60 & 193 \\
24 Jan. 2002 & MDM & $B G 38$ & 4 & 597 \\
30 May 2002 & DAO & $B$ & 30 & 39 \\
1 Jul. 2002 & MDM & $B G 38$ & 5 & 216 \\
3 Feb. 2003 & MDM & $B$ & 10 & 350 \\
22 May 2003 & ULTRACAM & $u^{\prime} g^{\prime} i^{\prime}$ & 1.3 & $\sim 750$ \\
24 May 2003 & ULTRACAM & $u^{\prime} g^{\prime} i^{\prime}$ & 1.3 & $\sim 2860$ \\
25 May 2003 & ULTRACAM & $u^{\prime} g^{\prime} i^{\prime}$ & 1.3 & $\sim 3780$ \\
19 Jun. 2003 & MDM & $B$ & 10 & 166 \\
4 May 2004 & ULTRACAM & $u^{\prime} g^{\prime} i^{\prime}$ & 1.3 & $\sim 13000$ \\
5 Apr. 2006 & UVic & clear $^{\prime}$ & 33 & 359 \\
\hline
\end{tabular}

Notes: Col. (1): observing date; Col. (2): used telescope where UVic indicates the automatic 0.5-m telescope of the Climenhage Observatory in Victoria, Canada, DAO stands for the 1.8-m telescope of the Dominion Astrophysical Observatory, MDM is the $1.3-\mathrm{m}$ telescope of the Michigan-Dartmouth-MIT Observatory in Arizona, and ULTRACAM are observations with this instrument at the WHT; Col. (3): used filter; Col. (4): integration time per observation; Col. (5): number of observations.

\section{Observations and reductions}

\subsection{Photometry}

Our photometric dataset consists of various observations taken on a number of telescopes. Table 1 lists an overview of our photometric datasets taken with the 1.3-m telescope of the Michigan-Dartmouth-MIT (MDM) Observatory (Arizona), with the 4.2-m William Herschel Telescope (WHT) on La Palma with ULTRACAM (Dhillon \& Marsh 2001), with the automatic 0.5-m telescope of the Climenhage Observatory in Victoria, Canada (referred to as UVic), and with the 1.8-m telescope of the Dominion Astrophysical Observatory (DAO) located in Victoria, Canada.

To reduce dead-time for the MDM observations, the CCD was binned $2 \times 2$, resulting in a scale of $1.02^{\prime \prime}$ per binned pixel, and only a subregion of $256 \times 256$ (binned) pixels was read. These observations were reduced with standard packages in the Image Reduction and Analysis Facility (IRAF) ${ }^{1}$. We derived differential photometry with respect to one comparison star $(\mathrm{RA}=$ 13:26:59.6, Dec $=+45: 33: 05, \mathrm{~J} 2000$ ) that is bright enough in the sparse field. The UVic and DAO observations were reduced with standard packages in IRAF.

1 IRAF is distributed by the National Optical Astronomy Observatories, which are operated by the Association of Universities for Research in Astronomy, Inc., under cooperative agreement with the National Science Foundation. 
Table 2. Log of spectroscopic observations.

\begin{tabular}{rllcrl}
\hline \hline $\begin{array}{l}\text { Date } \\
\text { (1) }\end{array}$ & $\begin{array}{l}\text { Tel } \\
(2)\end{array}$ & $\begin{array}{l}\lambda(\AA) \\
(3)\end{array}$ & $\begin{array}{l}T(\mathrm{~s}) \\
(4)\end{array}$ & $\begin{array}{l}\# \\
(5)\end{array}$ & $\begin{array}{l}R(\AA) \\
(6)\end{array}$ \\
\hline 11 May 1998 & TLS & $4300-5300$ & 900 & 21 & 0.14 \\
12-18 May 1998 & TLS & $5650-10000$ & 900 & 75 & 0.16 \\
19-22 Jan. 2002 & Modspec & $4250-7550$ & 120 & 62 & 3.5 \\
16 Feb. 2002 & Modspec & $4250-7550$ & 120 & 5 & 3.5 \\
19 Feb. 2002 & Modspec & $4250-7550$ & 120 & 10 & 3.5 \\
5-7 May 2002 & CCDS & $4180-5100$ & 300 & 18 & 3.2 \\
13 May 2002 & CCDS & $4180-5100$ & 360 & 1 & 3.2 \\
13 May 2002 & CCDS & $4180-5100$ & 600 & 1 & 3.2 \\
12 Jun. 2002 & MkIII & $4250-7550$ & 90 & 1 & 3.7 \\
13 Jan. 2004 & Modspec & $4250-7550$ & 180 & 1 & 3.5 \\
17-18 Jan. 2004 & Modspec & $4250-7550$ & 180 & 2 & 3.5 \\
6 Mar 2004 & Modspec & $4250-7550$ & 180 & 2 & 3.5 \\
30 Jan. 2006 & ISIS & $3000-8000$ & 180 & 1 & 5 \\
30 Jan. 2006 & ISIS & $3000-8000$ & 240 & 6 & 5 \\
15 May 2006 & DAO & $3500-5150$ & 720 & 6 & 4.8 \\
\hline
\end{tabular}

Notes. Col. (1): observing date; Col. (2): used spectrograph, where TLS indicates the "Karl Schwarzschild" Tautenburg Echelle Spectrograph mounted at the the $2 \mathrm{~m}$ telescope of the Thüringer Landessternwarte; Modspec stands for the $2.4 \mathrm{~m}$ Hiltner Telescope (MDM) Modular Spectrograph; CCDS is the $2.4 \mathrm{~m}$ Hiltner Telescope CCD Spectrograph; MkIII is for the $2.4 \mathrm{~m}$ Hiltner Telescope Mark III Spectrograph; and DAO stands for the Cassegrain Spectrograph at the $1.8 \mathrm{~m}$ of the DAO. Col. (3): wavelength range of the spectra; Col. (4): integration time per observation; Col. (5): number of observations; Col. (6): resolution as derived from the FWHM of the arc lines.

The ULTRACAM data were reduced with standard aperture photometry. Differential photometry was obtained with respect to two comparison stars located at RA $=13: 26: 28.08$, Dec $=+45: 33: 11.6(\mathrm{~J} 2000)$ and $\mathrm{RA}=13: 26: 39.2$, Dec $=$ $+45: 34: 56.1$ (J2000). The coordinates of DE CVn are RA = $13: 26: 53.2, \mathrm{Dec}=+45: 32: 46.1(\mathrm{~J} 2000)$.

\subsection{Spectroscopy}

A $\log$ of our spectroscopic data set is given in Table 2. The TLS echelle spectra are reduced with standard packages in IRAF. We could not correct the TLS spectra for flat fielding because the sky/twilight flat fields would introduce only more lines in the spectra. The wavelength calibration was done with $\sim 300$ lines in the $\mathrm{Th}-\mathrm{Ar}$ arc spectra with a root-mean-square residual of $\sim 0.0036 \AA$. We checked the stability of the spectrograph by using sky lines and these are not shifted between the observations. Because of the unavailability of sky background or flux standards, the echelle spectra are not sky subtracted or fluxcalibrated. These spectra were taken during grey time, so we do not expect problems with the background level. The MDM (using the Modspec, CCDS, MkIII spectrographs), DAO, and ISIS spectra were all reduced with standard packages in IRAF and these are, except for the DAO spectra, approximately flux calibrated (a seeing matched slit width was used, limiting the accuracy of the photometric calibration).

\section{Results}

\subsection{Eclipse light curves}

The first ephemeris of the primary eclipse in DE CVn is given in Robb \& Greimel (1997). We take the mid-eclipse times as the mid-point between the start of the ingress and the end of the

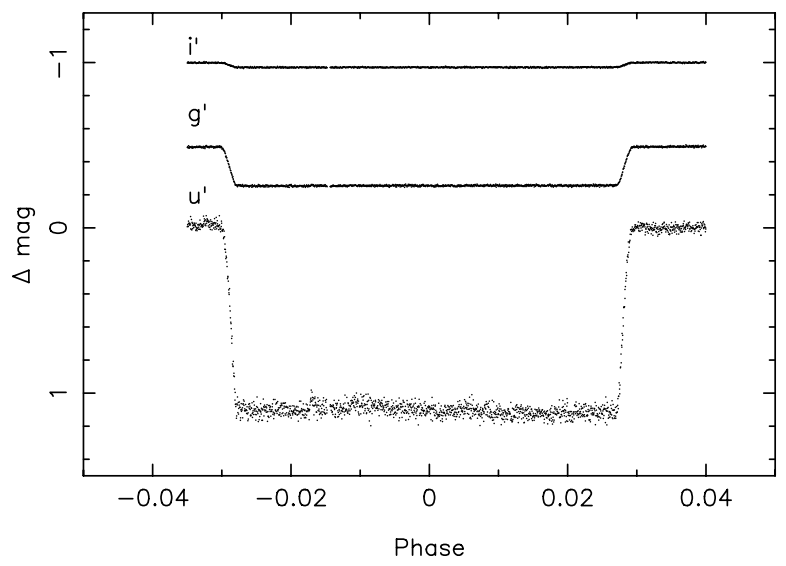

Fig. 1. Primary eclipse observed with ULTRACAM.

egress. From our photometric observations and the times of minima given in Tas et al. (2004) we have determined the ephemeris of the eclipse minima by fitting a straight line to the cycle numbers as derived from the ephemeris of Robb \& Greimel (1997):

$\mathrm{HJD}_{\min }=2452784.5533(1)+0.3641394(2) \times E$.

No significant aliases were found near this period. The uncertainties (last digits) are derived for $\Delta \chi^{2}=1$ when we scale the individual errors to obtain that the reduced $\chi^{2}=1$. The value of the orbital period is further confirmed by the radial velocity analysis described in Sect. 3.5. The availability of two eclipses separated by only two cycles $(-6109$ and -6107$)$ leaves no cycle count ambiguity. Two mid-eclipse times of Tas et al. (2452411.3156 and 2452412.4078 ) were rejected due to the large phase shifts $(\Delta \phi>0.01$, in contrast to an average scatter of 0.004 for all other eclipses) with respect to the updated ephemeris of Robb \& Greimel (1997), leaving 23 mid-eclipse times for determining the ephemeris. When calculating the phase difference with our new period, it turns out that these two data points indeed have a large phase difference $(\Delta \phi>0.01)$, so most probably the published times of the minima are incorrect. The times of the minima together with the cycle number and the corresponding time difference are given in Table 3.

We observed a primary eclipse simultaneously in $u^{\prime}, g^{\prime}$, and $i^{\prime}$ with ULTRACAM. These data show the largest eclipse depth of $1.11 \pm 0.04 \mathrm{mag}$ in $u^{\prime}$. The eclipse depths in $g^{\prime}$ and $i^{\prime}$ are $0.235 \pm 0.004$ and $0.028 \pm 0.004 \mathrm{mag}$, respectively, where we have fitted straight lines to the out-of-eclipse points and in eclipse points to derive these values. The difference is taken as the eclipse depth. The uncertainties are derived for $\Delta \chi^{2}=1$ when we scale the individual errors to obtain that the reduced $\chi^{2}=1$.

\subsection{Apparent magnitudes}

In Table 4 we give the magnitudes of DE CVn in and out of eclipse of the ULTRACAM data as obtained with the second comparison star as given in Sect. 2.1. Also the MDM magnitudes and the magnitudes of the comparison stars are given. The MDM photometry taken on January 21, 2001 was obtained near phase 0.7 , well outside the eclipse. The transformation to standard magnitudes was derived using observations of four Landolt standard fields. Except for $U-B$, the transformations derived from the standard stars all had scatter below $0.03 \mathrm{mag}$. For $U-B$ the scatter is below 0.08 mag.

We derived the $u^{\prime}, g^{\prime}$, and $i^{\prime}$ magnitudes of DE CVn in and out of eclipse in the ULTRACAM data of May 24, 2003. The 
Table 3. Times of mid-eclipse for the binary DE CVn. The numbers in parentheses are the uncertainties on the mid-eclipse times in the last digits.

\begin{tabular}{clr}
\hline \hline HJD-2450000 & \multicolumn{1}{c}{ cycle } & \multicolumn{1}{c}{$\Delta$ (HJD) } \\
\hline $550.9214(16)^{a}$ & -6134 & -0.00060 \\
$560.0243(16)^{a}$ & -6109 & -0.00118 \\
$560.7531\left(20^{a}\right)$ & -6107 & -0.00066 \\
$562.9374(22)^{a}$ & -6101 & -0.00120 \\
$570.9497(14)^{a}$ & -6079 & 0.00004 \\
$576.7749(14)^{a}$ & -6063 & -0.00100 \\
$578.9606(6)^{a}$ & -6057 & -0.00013 \\
$995.9003(18)^{b}$ & -4912 & -0.00010 \\
$1620.7628(15)^{b}$ & -3196 & -0.00089 \\
$2050.8125(16)^{b}$ & -2015 & 0.00012 \\
$2055.9103(7)^{c}$ & -2001 & -0.00003 \\
$2062.8289(7)^{b}$ & -1982 & -0.00008 \\
$2295.8782(4)^{d}$ & -1342 & -0.00003 \\
$2298.7914(1)^{d}$ & -1334 & 0.00006 \\
$2411.3156(1)^{e g}$ & -1025 & 0.00517 \\
$2412.4078(22)^{e g}$ & -1022 & 0.00495 \\
$2413.4958(4)^{e}$ & -1019 & 0.00053 \\
$2424.7839(6)^{c}$ & -988 & 0.00031 \\
$2456.8279(4)^{d}$ & -900 & 0.00004 \\
$2673.85488(14)^{d}$ & -304 & -0.00009 \\
$2705.5359(3)^{e}$ & -217 & 0.00079 \\
$2727.3837(4)^{e}$ & -157 & 0.00023 \\
$2784.553370(25)^{f}$ & 0 & 0.00000 \\
$2809.6788(1)^{d}$ & 69 & -0.00019 \\
$3830.7256(4)^{b}$ & 2873 & -0.00040 \\
\hline
\end{tabular}

Notes: ${ }^{a}$ Robb \& Greimel (1997); ${ }^{b}$ UVic photometry; ${ }^{c}$ DAO photometry; ${ }^{d}$ MDM photometry; ${ }^{e}$ Tas et al. (2004); ${ }^{f}$ ULTRACAM photometry; ${ }^{g}$ Not used for determining the ephemeris.

part just before the actual eclipse is taken for the out-of-eclipse magnitudes. All the ULTRACAM magnitudes were derived by using the two comparison stars in the field as mentioned in Sect. 2.1. Smith et al. (2002) values were used to derive the $u^{\prime}, g^{\prime}$, and $i^{\prime}$ magnitudes. The magnitudes for DE CVn were the same using either comparison star within $\sim 0.01 \mathrm{mag}$ in $g^{\prime}$ and $i^{\prime}$, and $\sim 0.02$ mag in $u^{\prime}$ ( $1 \sigma$ uncertainty).

\subsection{The nature of the components}

DE CVn has not been studied spectroscopically before. An average low resolution spectrum taken with the ISIS spectrograph on the WHT on La Palma is shown in Fig. 2. Clearly visible are the absorption bands of $\mathrm{TiO}$ indicating an M-type star. Emission lines of $\mathrm{H} \alpha, \mathrm{H} \beta, \mathrm{H} \gamma, \mathrm{H} \delta$, and $\mathrm{Ca}$ II $\mathrm{H} \& \mathrm{~K}$ are visible as well.

$\mathrm{DE} C \mathrm{C} n$ is a single-lined spectroscopic eclipsing binary. We do not see any spectral features of the white dwarf in the overall spectrum. The six low dispersion DAO spectra referred to in Table 2 were observed consecutively before, during, and after an eclipse of the white dwarf by the red dwarf. The sum of the two spectra taken during the eclipse were then subtracted from the sum of the two spectra taken immediately before the eclipse. The resultant smoothed spectrum is plotted in Fig. 2. Using the spectra taken after the eclipse resulted in a similar spectrum. The strong hydrogen absorption lines are typical of a DA white dwarf, and by visually comparing our WD spectrum with the ones in Wesemael et al. (1993), we come to a spectral type of DA7 \pm 0.5 , which corresponds to a temperature of $7500 \pm 1000 \mathrm{~K}$. The lack of residual Ca II K and narrow hydrogen emission lines gives us confidence that the subtraction was done correctly and the spectra did not need to be scaled.

To determine the characteristics of the two components we fit the averaged ISIS spectrum with a composite model consisting of a white dwarf and a red dwarf. We first corrected the spectra for the radial velocity variations as a function of phase before averaging the ISIS spectra. The comparison spectra that were used to fit the data consist of a white dwarf model spectrum with a hydrogen atmosphere and temperatures between 1500 and $17000 \mathrm{~K}$ (kindly made available to us by P. Bergeron: Bergeron et al. 1991, 1995). From the most likely white dwarf mass and radius as derived from the eclipse fitting in Sect. 3.8, we derive a surface gravity for the white dwarf of $\log g \sim 7.8$. Therefore we have used only white dwarf template spectra with surface gravity $\log g=7.5$. A red dwarf template (M0V to M6V in integer types) together with the corresponding absolute visual magnitude was taken from the library of Pickles (1998). We first scaled the individual spectra to $10 \mathrm{pc}$ before adding them together. We calculated the reduced $\chi^{2}$ of the fit to the average ISIS DE CVn spectrum for all the different model composite spectra to determine the nature of the components in this binary. The composition of the model spectrum with the lowest reduced $\chi^{2}$ is taken as the best combination. A more extended description of the fitting procedure will be given in a future paper.

For the fitting of DE CVn, we have excluded the wavelength regions around the emission lines of $\mathrm{H} \alpha, \mathrm{H} \beta, \mathrm{H} \gamma, \mathrm{H} \delta$, and $\mathrm{Ca}$ II $\mathrm{H} \& \mathrm{~K}$ and the earth atmosphere bands. The best fit consists of the combination of a red dwarf with spectral type M3V and a white dwarf with a temperature of $8000 \mathrm{~K}$, although the corresponding formal reduced $\chi^{2}$ is high (511). The second best fit has a $\Delta \chi^{2}=50$. When we take all the combinations with a $\chi^{2}<1000$, the spectral type of the red dwarf stays the same, while the temperature varies between 7000 and $9000 \mathrm{~K}$. Therefore we take an uncertainty on the temperature of $1000 \mathrm{~K}$. If we used $\log g=8.0$ instead of $\log g=7.5$, the spectral type of the secondary stayed the same, while the temperature changed to $10000 \pm 1500 \mathrm{~K}$. Combined with the temperature estimate from the eclipse (end of this section) and the difference spectrum (see above), we decided to use the results from the fitting with the $\log g=7.5$ models.

The spectrum of DE CVn together with the best fit is shown in Fig. 2. Consistent results are found when fitting the MDM spectra, which cover a shorter wavelength range. The discrepancy between the data and the fit of the spectrum is likely due to flux calibration errors, different intrinsic properties of the red dwarf such as metallicity, and the non-removal of telluric features in our spectra.

Another method to derive the secondary spectral type is by using the TiO5 index. Reid et al. (1995) have given the bestfit linear relation between the spectral type of a late-type mainsequence star and its TiO5 band strength:

$S_{\mathrm{p}}=-10.775 \times \mathrm{TiO} 5+8.2$

where TiO5 is the band strength as defined by $F_{\mathrm{w}} / F_{\text {cont }}$ with $F_{\mathrm{w}}$ the flux in the 7126-7135 $\AA$ region and $F_{\text {cont }}$ as the flux in the 7042-7046 A region. Using this definition we have calculated the TiO5 band strength and the corresponding spectral type for all the MDM spectra covering the wavelength range given above. The white dwarf contribution to this part of the spectrum is very small $(\leq 5 \%)$, so this will not affect the ratio. The corresponding average $S_{\mathrm{p}}$ is 2.17 , corresponding to spectral type M2.

From the single M3 spectrum of Pickles (1998) we have calculated the TiO5 band strength and corresponding spectral type 


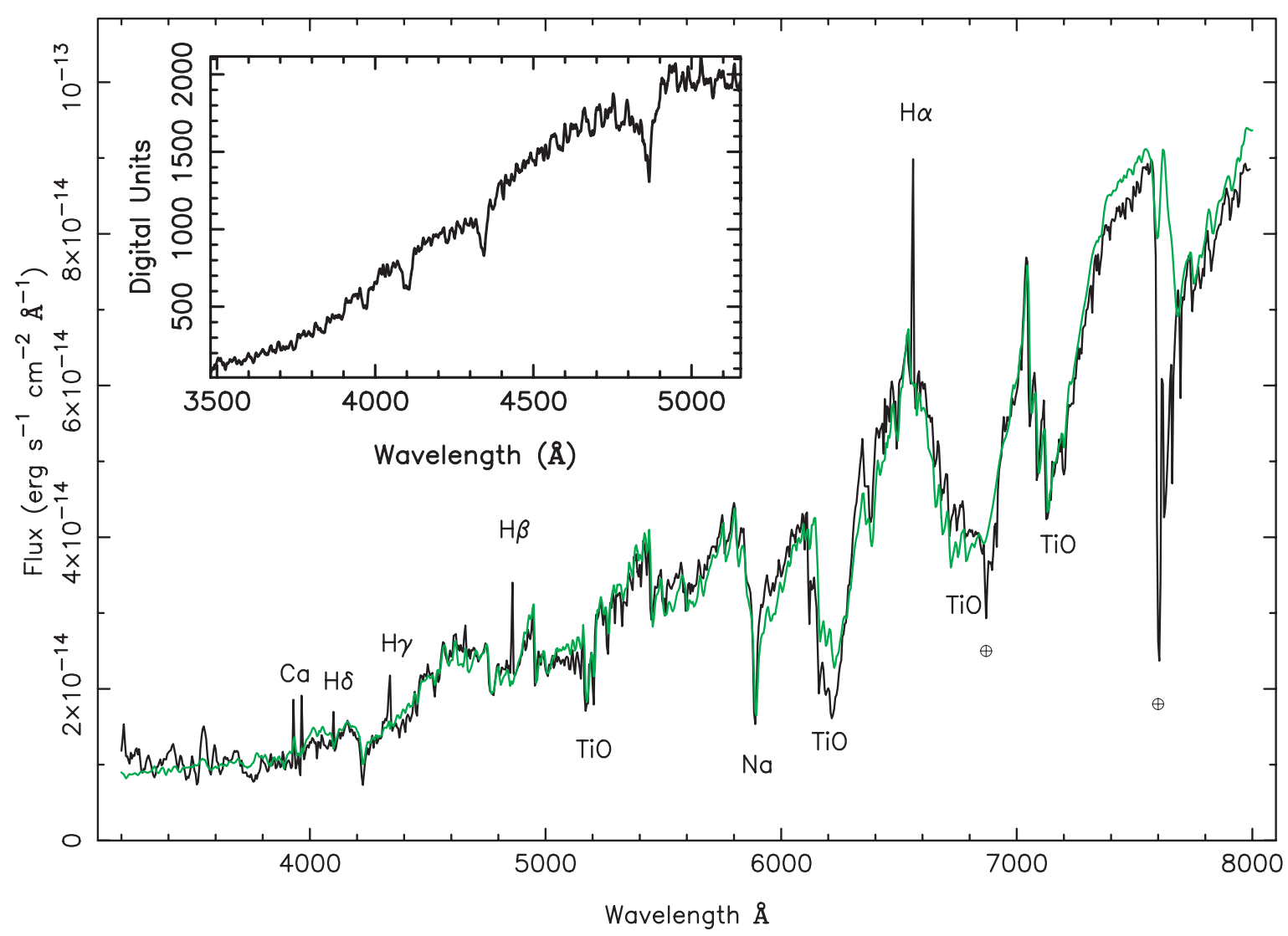

Fig. 2. A combined ISIS spectrum of DE CVn (black line) together with a composite template of an M3V star from Pickles (1998) and a DA white dwarf with a temperature of $8000 \mathrm{~K}$ and $\log g=7.5$ (grey/green line). Emission lines of $\mathrm{H} \alpha, \mathrm{H} \beta, \mathrm{H} \gamma$, and $\mathrm{H} \delta$ are visible in the spectrum as well as the Ca II H\&K emission lines and the TiO absorption bands of the M dwarf. No spectral line signatures of the primary are visible. The difference between the in and out-of-eclipse spectra shows the underlying white dwarf, which is plotted in the upper left corner.

Table 4. $u^{\prime}, g^{\prime}, i^{\prime}$ (ULTRACAM, May 24, 2003), and $U B V I$ (MDM) magnitudes of DE CVn and the two comparison stars with the uncertainty on the last digits in parentheses.

\begin{tabular}{lcccllll}
\hline \hline & $u^{\prime}$ & $g^{\prime}$ & \multicolumn{1}{c}{$i^{\prime}$} & \multicolumn{1}{c}{$V$} & \multicolumn{1}{c}{$U-B$} & $B-V$ & \multicolumn{1}{c}{$V-I$} \\
\hline DE CVn in eclipse & $16.43(1)$ & $13.74(1)$ & $11.65(2)$ & - & - & - & - \\
DE CVn out-of-eclipse & $15.31(1)$ & $13.50(1)$ & $11.62(2)$ & $12.908(2)$ & $0.070(15)$ & $1.263(5)$ & $2.244(2)$ \\
Comparison 1 & $15.16(1)$ & $13.22(1)$ & $12.29(2)$ & - & - & - & - \\
Comparison 2 & $15.77(1)$ & $13.61(1)$ & $13.34(2)$ & $13.418(2)$ & $0.155(13)$ & $0.708(5)$ & $0.784(4)$ \\
\hline
\end{tabular}

as well. This gives a strength of 0.55 and a spectral type of 2.27 . By comparing the value of the M3 spectrum with the intrinsic variation as seen in Fig. 2 of Reid et al. (1995), we see that this value is consistent with the values derived for DE CVn. From this we see that the different methods are consistent with a red dwarf of spectral type M3 as used by Pickles (1998).

From the apparent magnitudes of DE CVn in eclipse and outside eclipse, we can derive the colour of the unseen white dwarf that is being eclipsed. This gives $\left(u^{\prime}-g^{\prime}\right)_{\mathrm{WD}}=0.52 \pm 0.01$ and $\left(g^{\prime}-i^{\prime}\right)_{\mathrm{WD}}=-0.26 \pm 0.02$. By comparing this with the values from the white dwarf models the $u^{\prime}-g^{\prime}$ colour indicates a temperature of 7000-9000 K, while the $g^{\prime}-i^{\prime}$ colour indicates $6000-8000 \mathrm{~K}$. This is fully consistent with the spectral modelling.

\subsection{Spectral line variations}

The spectra show emission lines of hydrogen up to $\mathrm{H} 10$ and $\mathrm{Ca}$ II $\mathrm{H} \& \mathrm{~K}$ emission. We have searched the normalised TLS echelle spectra for spectral lines showing radial velocity variations, either in phase with the Balmer and Ca II H\&K lines or in anti-phase. All lines identified are listed in Table 5 together with their equivalent widths $(E W)$. No lines were seen to move in anti-phase with the Balmer lines. The lines that do not have an $E W$ value are blended with sky lines so that we cannot derive an accurate value for the $E W$. The $E W$ of the bluest $\mathrm{H}$ lines were measured in the average ISIS spectrum for which we first removed the phase shifts in the individual spectra.

\subsection{Radial velocity curve}

Radial velocities of the $\mathrm{H} \alpha$ lines in the TLS spectra were determined by fitting a single Gaussian line profile and a first-order polynomial to the emission line and the surrounding continuum. The radial velocities of the $\mathrm{H} \beta$ and $\mathrm{H} \gamma$ lines in the TLS spectra were also measured in this way. The typical uncertainties on the radial velocities of the TLS spectra for $\mathrm{H} \alpha, \mathrm{H} \beta$, and $\mathrm{H} \gamma$ are $\sim 0.3 \mathrm{~km} \mathrm{~s}^{-1}, \sim 0.4 \mathrm{~km} \mathrm{~s}^{-1}$, and $\sim 0.7 \mathrm{~km} \mathrm{~s}^{-1}$, respectively. The MDM spectra were cross-correlated with an M dwarf spectrum 
Table 5. Identified lines in the TLS echelle spectra and the ISIS $\left(^{a}\right)$ spectrum together with their equivalent widths.

\begin{tabular}{|c|c|c|c|c|c|c|c|}
\hline $\begin{array}{l}\lambda_{\mathrm{obs}}(\AA) \\
(1)\end{array}$ & $\begin{array}{l}\lambda(\AA) \\
(2)\end{array}$ & $\begin{array}{l}\text { Element } \\
\text { (3) }\end{array}$ & $\begin{array}{l}E W(\AA) \\
(4)\end{array}$ & $\begin{array}{l}\lambda_{\text {obs }}(\AA) \\
(1)\end{array}$ & $\begin{array}{l}\lambda(\AA) \\
(2)\end{array}$ & $\begin{array}{l}\text { Element } \\
\text { (3) }\end{array}$ & $\begin{array}{l}E W(\AA) \\
(4)\end{array}$ \\
\hline $3797.20^{a}$ & 3797.91 & H 10 & -0.9 & 6764.56 & 6764.13 & Fe I & 0.3 \\
\hline $3832.45^{a}$ & 3835.397 & H9 & -1.8 & 6768.72 & 6768.65 & Ti I & 0.4 \\
\hline $3887.33^{a}$ & 3889.05 & H 8 & -2.1 & 6773.05 & 6772.36 & Ni I & 0.3 \\
\hline $3932.47^{a}$ & 3933.67 & Ca II K & -6.2 & 6777.97 & 6777.44 & $\mathrm{Fe} I$ & 0.3 \\
\hline $3968.10^{a}$ & 3970.874 & $\mathrm{H} \epsilon+\mathrm{Ca}$ II H & -7.5 & 6806.81 & 6806.85 & $\mathrm{Fe} I$ & 0.3 \\
\hline $4101.26^{a}$ & 4101.735 & $\mathrm{H} \delta$ & -2.8 & 6815.83 & 6815 & $\mathrm{TiO}$ & 0.5 \\
\hline $4340.00^{a}$ & 4340.465 & $\mathrm{H} \gamma$ & -3.7 & 6921.45 & 6920.16 & $\mathrm{Fe} I$ & 0.3 \\
\hline $4860.63^{a}$ & 4861.327 & $\mathrm{H} \beta$ & -4.1 & 7055.51 & 7054 & $\mathrm{TiO}$ & 1.5 \\
\hline 5094.51 & 5093.646 & Fe II & 0.4 & 7060.87 & 7059.941 & Ba I & 0.6 \\
\hline 5099 & 5098.703 & $\mathrm{Fe} I$ & $<0.2$ & 7088.90 & 7088 & $\mathrm{TiO}$ & 1.0 \\
\hline 5164 & $5163.940 / 5164.70$ & $\mathrm{Fe}$ II/Fe I & $<0.2$ & 7126.37 & 7126 & $\mathrm{TiO}$ & 1.3 \\
\hline 5167 & 5167.000 & $\mathrm{TiO}$ & $<0.2$ & 7148.12 & 7148.147 & $\mathrm{CaI}$ & 0.4 \\
\hline 5210 & 5209.900 & $\mathrm{Fe} I$ & $<0.2$ & 7326.21 & 7326.146 & $\mathrm{CaI}$ & 0.6 \\
\hline 5230 & 5229.857 & Fe I & $<0.2$ & 7400.12 & $7400.87 / 7400.23$ & $\mathrm{Fe} \mathrm{I/Cr} \mathrm{I}$ & 0.2 \\
\hline 5240 & 5240.000 & $\mathrm{TiO}$ & $<0.2$ & 7462.33 & 7461 & $\mathrm{Fe}_{\mathrm{I}}$ & 0.3 \\
\hline 6102.78 & 6101.100 & K IV & 1.3 & 7590 & 7589 & $\mathrm{TiO}$ & blended \\
\hline 6122.01 & 6122.219 & $\mathrm{CaI}$ & 1.8 & 7666 & 7666 & $\mathrm{TiO}$ & blended \\
\hline 6157.08 & 6159 & $\mathrm{TiO}$ & 0.9 & 7674 & 7671 & $\mathrm{TiO}$ & blended \\
\hline 6381.49 & 6384 & $\mathrm{TiO}$ & 0.8 & 7701 & 7704 & $\mathrm{TiO}$ & blended \\
\hline 6388.01 & 6388.410 & $\mathrm{Fe} I$ & 0.8 & 7800.24 & 7800.00 & Si I & 0.3 \\
\hline 6393.13 & $6391.214 / 6391.51$ & Mn I/Ti II & 0.4 & 8047.31 & 8047.6 & $\mathrm{Fe} I$ & 0.3 \\
\hline 6421.11 & 6421.355 & $\mathrm{Fe} I$ & 0.3 & 8182.88 & 8183.256 & $\mathrm{Na} \mathrm{I}$ & 1.4 \\
\hline 6439.01 & 6439.073 & $\mathrm{CaI}$ & 0.9 & 8194.69 & $8194.79 / 8194.82$ & $\mathrm{NaI}$ & 1.4 \\
\hline 6449.78 & 6450.854 & $\mathrm{BaI}$ & 1.0 & 8325 & 8323.428 & $\mathrm{H}$ & blended \\
\hline 6462.64 & 6462.566 & $\mathrm{Ca} \mathrm{I}$ & 1.2 & 8362 & 8361.77 & He I & blended \\
\hline 6471.56 & 6472.34 & Sm II & 0.5 & 8379 & 8377.6 & $\mathrm{Ne} I$ & blended \\
\hline 6494.22 & 6493.78 & $\mathrm{CaI}$ & 1.0 & 8382 & 8382.23 & $\mathrm{Fe} I$ & blended \\
\hline 6499.39 & 6498.759 & $\mathrm{Ba} \mathrm{I}$ & 0.5 & 8387 & 8387.78 & $\mathrm{Fe} \mathrm{I}$ & blended \\
\hline 6562.75 & 6562.76 & $\mathrm{H} \alpha$ & -6.7 & 8411 & 8412.97 & $\mathrm{Fe} \mathrm{I}$ & blended \\
\hline 6572.81 & 6572.781 & $\mathrm{CaI}$ & 0.5 & 8424 & $8424.14 / 8424.78$ & $\mathrm{Fe} \mathrm{I} / \mathrm{O} \mathrm{I}$ & blended \\
\hline 6593.79 & 6595.326 & $\mathrm{Ba} \mathrm{I}$ & 0.5 & 8662.03 & 8661.908 & $\mathrm{Fe} \mathrm{I}$ & 1.0 \\
\hline 6626.22 & 6627.558 & Fe I & 0.3 & 8674.98 & 8674.751 & $\mathrm{Fe} I$ & 0.3 \\
\hline 6651.83 & 6651 & $\mathrm{TiO}$ & 0.4 & 8688.44 & 8688.633 & $\mathrm{Fe} I$ & 0.5 \\
\hline 6685.09 & 6681 & $\mathrm{TiO}$ & 0.9 & 8806.51 & 8805.21 & $\mathrm{Fe} I$ & 0.4 \\
\hline 6719.14 & 6715 & $\mathrm{TiO}$ & 1.3 & 8824.21 & 8820.45 & O I & 0.6 \\
\hline 6760.15 & 6760.61 & $\mathrm{Fe} I$ & 0.4 & & & & \\
\hline
\end{tabular}

Notes: Col. (1): wavelength of the line as measured in the echelle spectra; Col. (2): wavelength corresponding to the element that we identify this line with (Col. (3)); Col. (4): equivalent width of the line. Lines without an equivalent width measurement are blended with sky lines. The typical uncertainty on the $E W$ is $\sim 0.1 \AA$.

over the 6000-6500 А range. The uncertainties for these crosscorrelated radial velocities are $\sim 2.2 \mathrm{~km} \mathrm{~s}^{-1}$.

To derive the semi-amplitude of the radial velocity variations of the secondary and the systemic velocity we use the measurements of the $\mathrm{H} \alpha$ line in the TLS spectra because these are the most accurate measurements. There is no spectral line feature of the primary star visible in the spectra, so therefore we cannot derive the semi-amplitude of the white dwarf. We have used the function

$f(\phi)=\gamma+K_{2} \sin \left(2 \pi\left(\phi-\phi_{0}\right)\right)$,

where $K_{2}$ is the semi-amplitude of the secondary star and $\gamma$ the systemic velocity in $\mathrm{km} \mathrm{s}^{-1}$ to fit the data. The best fit gives $\gamma=$ $-7.5 \pm 3 \mathrm{~km} \mathrm{~s}^{-1}, K_{2}=166 \pm 4 \mathrm{~km} \mathrm{~s}^{-1}$, and $\phi_{0}=-0.004 \pm 0.004$ for fitting only the TLS $\mathrm{H} \alpha$ variations. The uncertainties are derived from $\Delta \chi^{2}=1$ when the reduced $\chi^{2}=1$. The phase of the radial velocity curve is consistent with the time of mid-eclipse. The fit is shown in Fig. 3 together with the measured radial velocity variations of $\mathrm{H} \alpha$. The residuals of $\mathrm{H} \alpha, \mathrm{H} \beta, \mathrm{H} \gamma$, and the $\mathrm{TiO}$ absorption features are shown Fig. 4. When fitting for an eccentric orbit, we find an insignificant eccentricity of $e=0.02 \pm 0.02$, so assuming a circular orbit is justified. No differences in the radial velocity curves of $\mathrm{H} \beta, \mathrm{H} \gamma$, and $\mathrm{TiO}$ absorption with respect to the $\mathrm{H} \alpha$ observations are observed.

\subsection{Irradiation}

In close binary systems we may expect to see irradiation on the red dwarf due to heating of the close white dwarf. To investigate this effect we have measured the variations of the $E W$ of the $\mathrm{H} \alpha$ line. In Fig. 5 we show the variations of the $E W$ of this line folded on the orbital period. A typical error bar is shown in the bottom left corner of the figure. There is considerable variation of the $\mathrm{H} \alpha$ line, but this does not coincide with the time scale of the orbital period. During the eclipse, the $E W$ is larger compared to the one outside the eclipse. If the $\mathrm{H} \alpha$ emission originates in the atmosphere of the secondary due to irradiation of the white dwarf, we would expect to see variations in the strength corresponding to the time scale of the orbital period.

In the similar system RR Cae, consisting of a white dwarf of $7000 \mathrm{~K}$ and an M6 or later secondary, a similar effect of a larger $E W$ during eclipse was seen (Bruch 1999). This was 


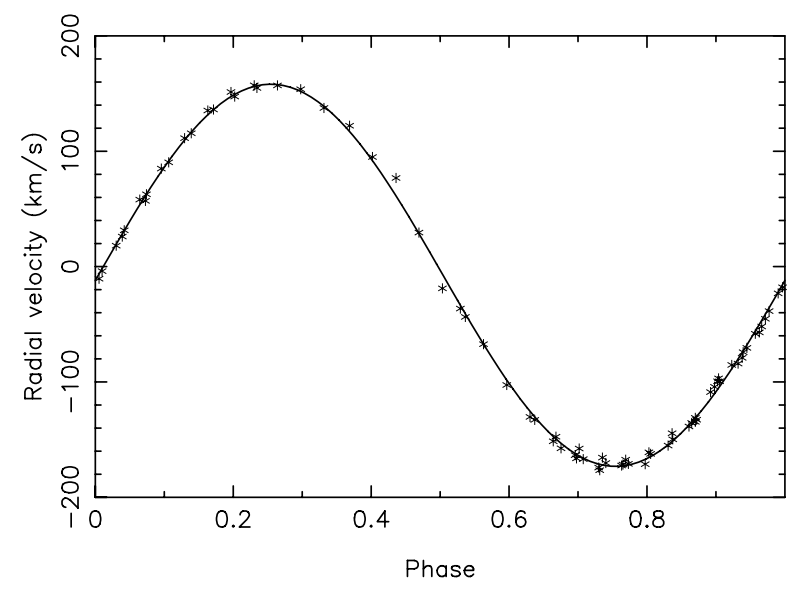

Fig. 3. Radial velocity measurements for the $\mathrm{H} \alpha$ lines. The solid line is the best fit to these velocities, as discussed in Sect. 3.5. The uncertainties on the points are smaller than the symbols.

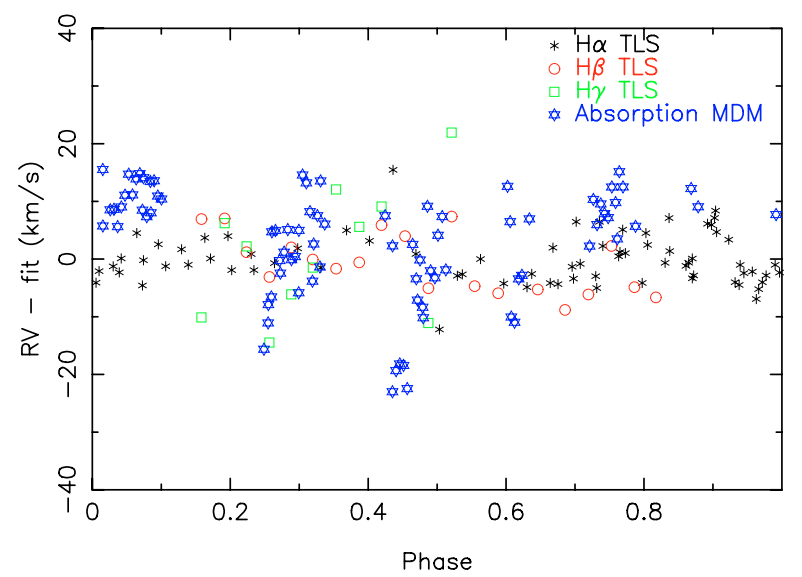

Fig. 4. Residuals of the $\mathrm{H} \alpha, \mathrm{H} \beta, \mathrm{H} \gamma$, and the MDM absorption lines as compared with the best-fit sinusoid to the $\mathrm{H} \alpha$ data.

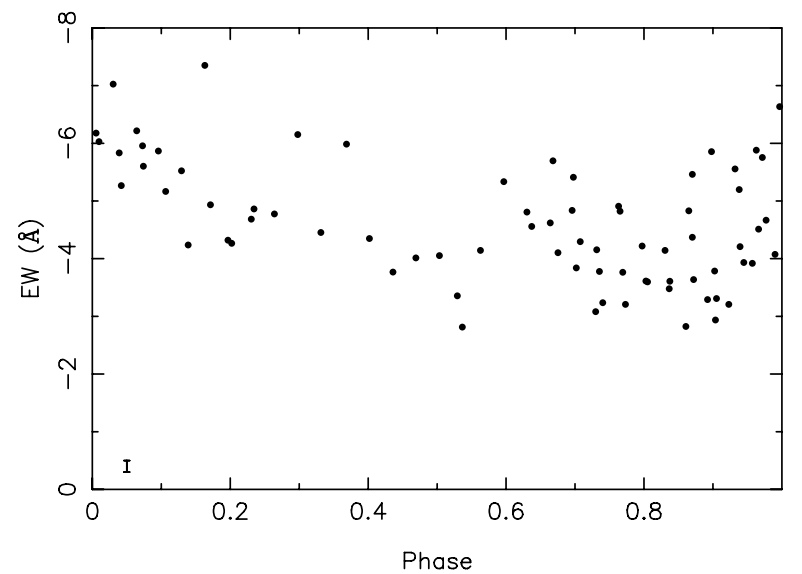

Fig. 5. The equivalent width of the $\mathrm{H} \alpha$ line in the TLS spectra. The variations are folded on the orbital period of DE CVn. A typical error bar is shown in the bottom left corner.

contributed to the emission lines being intrinsic to the secondary itself (Bruch 1999). In analogy, we therefore conclude that the $\mathrm{H} \alpha$ emission in DE CVn is also due to activity in the chromosphere of the M dwarf in contrast to being caused by irradiation. Furthermore, we can rule out $\mathrm{H} \alpha$ emission emerging from an

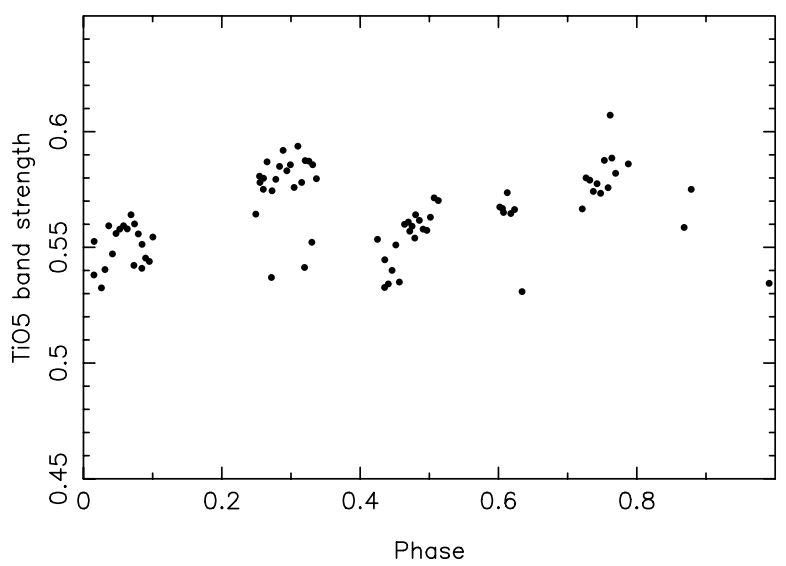

Fig. 6. TiO5 band strength of the MDM spectra as a function of orbital phase. The uncertainty on each point is smaller than the symbol.

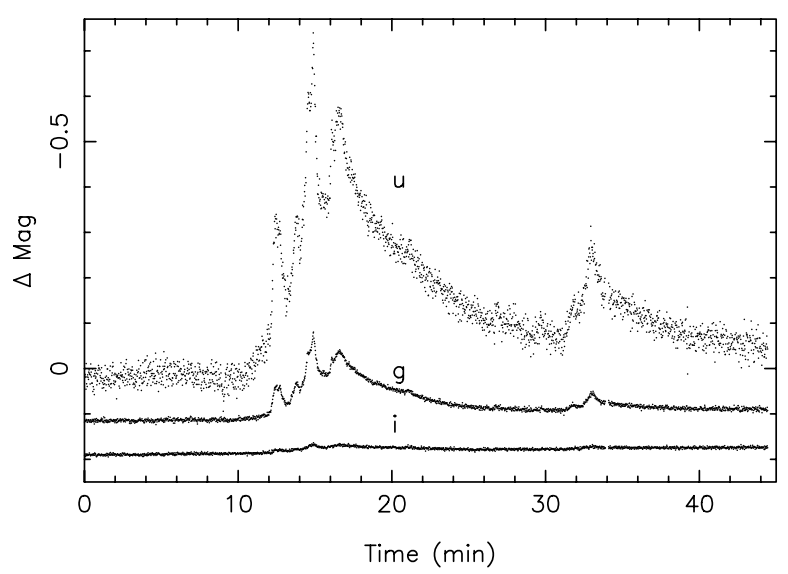

Fig. 7. A flare of DE CVn observed with ULTRACAM.

accretion disc because of its regular radial velocity profile and the single peaked lines.

If the secondary star is irradiated by the white dwarf, we would also expect a variation of the TiO5 band strength (which is discussed in Sect. 3.3) with respect to the orbital period due to heating in the atmosphere of the secondary. We see variations (see Fig. 6), but not with the orbital period. Thus also in this case, we conclude that the variations are intrinsic to the secondary.

\subsection{Flare}

During one of the ULTRACAM observing runs (25 May 2003), a flare was observed in all three bands starting at about 23:23 UT. This part of the light curve is shown in Fig. 7. The observed part of the flare lasted $\sim 39 \mathrm{~min}$. The flare has a complex structure with several peaks close together and a rebrightening during the decay part after the first peaks. Unfortunately the observation ended before DE CVn had returned to the quiescent (pre-flare) luminosity (most clearly seen in the $u^{\prime}$ band). From the observed count rates during quiescence and the average count rate during the observed part of the flare, we calculated that DE CVn increased in brightness on average by $20 \%, 5 \%$, and $1 \%$ of the quiescent flux (so white dwarf + red dwarf) during this flaring period in $u^{\prime}, g^{\prime}$, and $i^{\prime}$, respectively.

To model the flare, we have taken a blackbody spectrum added to the best-fitted template spectrum (see Sect. 3.3). A blackbody might not be a good approximation, but it is used in other analyses of flare stars as well (de Jager et al. 1986, 1989). 


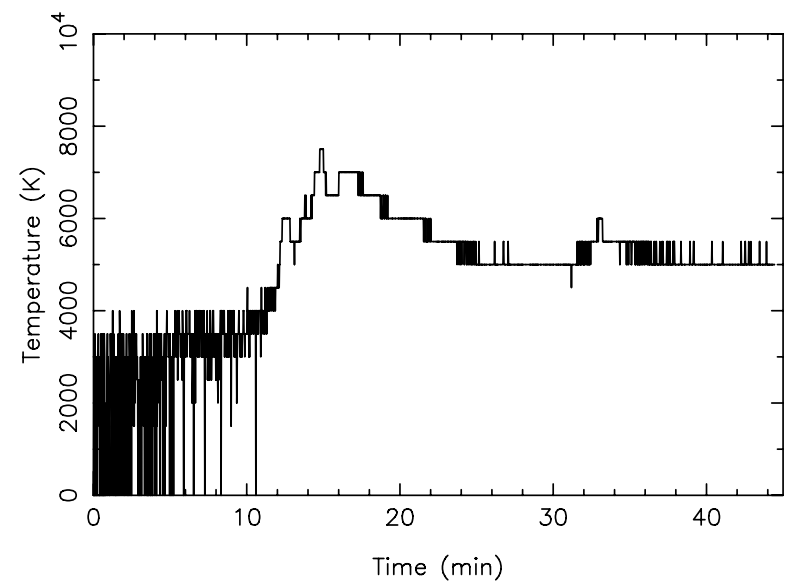

Fig. 8. Temperature variation of the flaring area during the flaring period. The flaring area has a radius of $0.038 R_{\odot}$.

We have assumed a constant (but not pre-determined) flaring area that is facing us with only temperature variations over time. We have assumed temperatures between 0 and $20000 \mathrm{~K}$ in steps of $200 \mathrm{~K}$ and a constant radius for the flaring area.

For every point in time we have taken the three corresponding observed magnitudes. We have derived the $u^{\prime}, g^{\prime}$, and $i^{\prime}$ magnitudes for our model by convolving the filter curves with our model spectrum for each temperature. For each point in time we have calculated the reduced $\chi^{2}$ for each temperature and we have taken the temperature with the lowest reduced $\chi^{2}$ for this point. After this, the average reduced $\chi^{2}$ over all the time points was calculated.

We performed this method for several constant flaring areas with radii between $0.010 R_{\odot}$ and $0.053 R_{\odot}$ with steps of $0.001 R_{\odot}$. The result with the lowest average reduced $\chi^{2}$ has a radius $0.038 R_{\odot}$ and the temperature variations are shown in Fig. 8. The area that flares corresponds to $0.9 \%$ of the visible stellar disc of the red dwarf.

\subsection{System parameters}

Because spectral features from the white dwarf are not visible we can only measure the radial velocity curve of the secondary star. The inclination of the system is constrained by the eclipses in the photometric light curve. We have fitted the ULTRACAM $g^{\prime}$ light curve with a simple model light curve that results from using two overlapping circles representing a white dwarf and a red dwarf that is (partly) obscuring the white dwarf (see Van Ham et al. 2007, submitted). It is assumed that the light intensity is proportional to the visible part of the white dwarf. We have used the light curve in counts scaled to range between 1 (out of eclipse) and 0 (in eclipse). This way we only derive the radii of the two stars with respect to the orbital separation $\left(a\right.$, i.e. $R_{\mathrm{WD}} / a$ and $\left.R_{\mathrm{RD}} / a\right)$.

Limb darkening of the white dwarf could play a role. When we include limb darkening in our model the white dwarf radius increased by a few per cent. This is negligible with respect to the uncertainty in the inclination so therefore we have neglected the effect of limb darkening in our method.

From our model we obtain the combinations of the white dwarf and red dwarf radii with respect to the orbital separation for inclinations between $75^{\circ}$ and $90^{\circ}$ with a step size of $1^{\circ}$. At lower inclinations we find that the red dwarf either fills its Roche lobe or is larger than its Roche lobe. As there is no evidence for mass transfer in the system, the red dwarf must be smaller than its Roche lobe. The reduced $\chi^{2}$ for these fits to the eclipse light curve are not significantly different, illustrating the fact that another constraint on the system is required for a unique solution.

The semi-amplitude of the radial velocity variations of the secondary is derived from the spectra in Sect. 3.5. The spectral type of the secondary is determined in Sect. 3.3 by fitting model spectra to the spectrum of DE CVn. These parameters are taken as known input parameters in our two-circle model.

From the spectral type of the secondary and assuming zeroage main-sequence masses and temperatures, the mass of the red dwarf should be between 0.3 and $0.5 M_{\odot}$ (see, e.g., Kirkpatrick et al. 1991). For every possible inclination, we input the mass of the secondary in steps of $0.01 M_{\odot}$ between these values. Furthermore, we use Kepler's third law and the mass-radius relation for white dwarfs from Eggleton as quoted by Verbunt \& Rappaport (1988):

$$
\begin{aligned}
R_{\mathrm{WD}}= & 0.0114 \cdot\left(\left(\frac{M_{\mathrm{WD}}}{M_{\mathrm{CH}}}\right)^{-\frac{2}{3}}-\left(\frac{M_{\mathrm{WD}}}{M_{\mathrm{CH}}}\right)^{\frac{2}{3}}\right)^{\frac{1}{2}} \\
& \times\left(1+3.5 \cdot\left(\frac{M_{\mathrm{WD}}}{M_{\mathrm{P}}}\right)^{-\frac{2}{3}}+\left(\frac{M_{\mathrm{WD}}}{M_{\mathrm{P}}}\right)^{-1}\right)^{-\frac{2}{3}},
\end{aligned}
$$

where $R_{\mathrm{WD}}$ is in solar radii, $M_{\mathrm{WD}}$ in solar masses, $M_{\mathrm{CH}}=$ $1.44 M_{\odot}$, and $M_{\mathrm{P}}$ is a constant whose numerical value is $0.00057 M_{\odot}$.

For every inclination we obtain a value of $R_{\mathrm{WD}} / a$ from the eclipse fitting. By combining Kepler's third law and Eq. (4) we derive the mass and radius of the white dwarf for every inclination and secondary mass. With the radius of the white dwarf and the result of the eclipse fitting we derive the orbital separation and the radius of the secondary star.

By assuming a circular orbit, which is justified by the radial velocity curve, we can constrain the inclination by using the mass function given in Eq. (5) to derive the semi-amplitude of the red dwarf for every inclination and secondary mass, and the system parameters derived from the procedure described above. With these parameters and assuming a circular orbit we can use the following function for the mass function:

$$
\frac{M_{1}^{3} \sin ^{3} i}{\left(M_{2}+M_{1}\right)^{2}}=\left(1.0361 \times 10^{-7}\right) K_{2}^{3} P
$$

with $M_{1,2}$ in $M_{\odot}, K_{2}$ in $\mathrm{km} \mathrm{s}^{-1}$ and $P$ in days.

We take a $3 \sigma$ uncertainty on the measured semi-amplitude of the radial velocity variation of the secondary and compared these with the semi-amplitudes for each possible solution. We selected only those solutions that satisfied the radial velocity criterion. This constrains the inclination of DE CVn to $\geq 82^{\circ}$. The combined radial velocity and eclipse constraints lead to system parameters where the mass of the white dwarf is between 0.48 and $0.65 M_{\odot}$ and the radius between 0.0117 and $0.0140 R_{\odot}$. The red dwarf has a mass between 0.30 and $0.50 M_{\odot}$ and a radius between 0.36 and $0.51 R_{\odot}$. The orbital separation of the system is between 2.00 and $2.25 R_{\odot}$.

By comparing our white dwarf radii with the corresponding masses for carbon core or oxygen core white dwarfs from Panei et al. (2000), our minimum and maximum mass for the white dwarf would be at most $5 \%$ larger. Furthermore, all the red dwarf masses that we used in deriving the system parameters are possible solutions. In our ULTRACAM data set, we see hints for small out-of-eclipse light curve variations and variations between the 
different observations, but this data set does not cover the complete orbital period. In our eclipse fitting procedure, we only use the section between phases -0.035 and 0.04 , so here the effect of these variations can be neglected.

\subsection{Space velocity and distance}

The fitting procedure described in Sect. 3.3 also determines a distance to DE CVn of $28 \pm 1 \mathrm{pc}$. The proper motion is known as well (see Sect. 1), and the systemic velocity is derived from fitting the radial velocity curve in Sect. 3.5. Johnson \& Soderblom (1987) give the equations to calculate the space velocities $(U$, $V, W)$ and we use $\left(U_{\odot}, V_{\odot}, W_{\odot}\right)=(10.00 \pm 0.36,5.25 \pm 0.62$, $7.17 \pm 0.38)$ in $\mathrm{km} \mathrm{s}^{-1}$ as the velocity of the Sun with respect to the local standard of rest as given by Dehnen \& Binney (1998). The space velocities of DE CVn, defined as

$(u, v, w)=(U, V, W)-\left(U_{\odot}, V_{\odot}, W_{\odot}\right)$

are $(u, v, w)=(-15.8 \pm 0.4,-40.7 \pm 1.4,-3.3 \pm 2.8) \mathrm{km} \mathrm{s}^{-1}$. $U$ is defined as positive in the direction of the galactic center, $V$ is positive in the direction of the galactic rotation, and $W$ is positive in the direction of the galactic North Pole. The derived space velocities for DE CVn are consistent with being a thin disc object (Fuhrmann 2004).

\section{Discussion and conclusions}

\subsection{Binary parameters}

We have analysed various spectroscopic and photometric observations of the eclipsing binary DE CVn. The light curves show total eclipses of the primary by the secondary star. The low resolution average ISIS spectrum is fit with model composite spectra to determine the spectral type of the $\mathrm{M}$ dwarf and the temperature of the white dwarf. The best fit is derived for an M3V secondary star and the temperature of the white dwarf (with $\log g=7.5$ ) of $8000 \pm 1000 \mathrm{~K}$. This is consistent with the temperature estimates derived from the eclipse depths (Sect. 3.2) and difference spectrum (Sect. 3.3), and the spectral type from the TiO5-index (Sect. 3.3).

From the echelle spectra taken with the TLS we have obtained radial velocity curves of $\mathrm{H} \alpha, \mathrm{H} \beta$, and $\mathrm{H} \gamma$, and also $\mathrm{TiO}$ absorption bands from the MDM spectra. These curves show a semi-amplitude for the secondary of $166 \pm 4 \mathrm{~km} \mathrm{~s}^{-1}$. Combining the eclipse constraints with the radial velocity amplitude results in a set of solutions satisfying the constraints as described in Sect. 3.8. Determining the most likely value with an uncertainty on it is very complex. Due to the interdependency of the relations used, calculating the uncertainties is not straightforward. As an example, we show the histogram of the possible solutions for the white dwarf mass in Fig. 9, which shows an asymmetrical distribution. The average or the mean does not coincide with the peak of the distribution. We take the most likely value as the highest point in the distribution. To determine the uncertainty on the most likely value, we have taken the value for which $68 \%$ of the solutions are enclosed around the most likely value. The difference between these values is taken as our uncertainty. We have done this separately for each side of the distribution. For the white dwarf mass and radius this resulted in $0.51_{-0.02}^{+0.06} M_{\odot}$ and $0.0136_{-0.0002}^{+0.0008} R_{\odot}$. In the same way, the distributions of the red dwarf mass and radius result in $0.41 \pm 0.06 M_{\odot}$ and $0.37_{-0.007}^{+0.06} R_{\odot}$, respectively. The orbital separation is $2.07_{-0.04}^{+0.09} R_{\odot}$ and the inclination is $86_{-2}^{+3} \circ$. These most likely values are consistent with each other within the uncertainties.

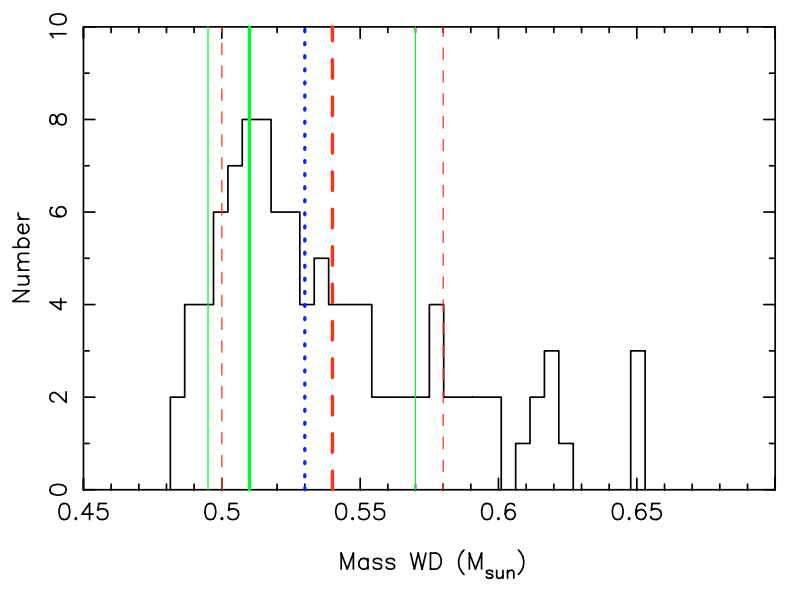

Fig. 9. Distribution of the possible solutions for the mass of the white dwarf. The thick solid line (green/grey) is the most likely value that we have used. The thin solid lines (green/grey) are the values between which $68 \%$ of the possible solutions are located. The thick dashed line (red/darker grey) is the average value with the standard deviation in thin dashed lines (red/darker grey). The median of the distribution is shown as the dotted line (blue/dark grey).

\subsection{Progenitor}

From the current small orbital separation we conclude that there was most probably a CE phase in the past in which the orbit has shrunken significantly. At the onset of the CE phase, the initially more massive star has evolved to a star with giant dimensions. We assume that the core of the giant star at the start of the CE phase had the same mass as the present-day white dwarf. This can be used to reconstruct the evolution of DE CVn to find the possible progenitors.

To do this we take single main-sequence stars with masses of 1 to $8 M_{\odot}$ in steps of $0.1 M_{\odot}$. We evolve these stars with the method described in Hurley et al. (2000). When the core mass $\left(M_{\mathrm{c}}\right)$ of the stars has reached the mass of the white dwarf that we observe in DE CVn $\left(0.51 M_{\odot}\right)$, the evolution is stopped. Then we check if the radius of the star at this point $\left(R_{i}\right)$ corresponds to the largest radius during the evolution up to this point. We assume that the star fills its Roche lobe in the giant phase.

The most likely mass of the white dwarf of DE CVn falls in a mass range where many progenitor stars cannot fill their Roche lobe with this core mass. The reason is that on the first giant branch their core grows to $\sim 0.48 M_{\odot}$, when the helium flash happens. Then the star contracts while the core mass still increases. When the star expands again to ascend the asymptotic giant branch (AGB), the core mass has become larger than the most likely white dwarf mass in DE CVn. However, this is very sensitive to the core mass: for a core mass of $0.51 M_{\odot}$ we find only one progenitor, while for $0.52 M_{\odot}$ we find six. We therefore use $0.52 M_{\odot}$, as a compromise between staying close to the most likely mass yet allowing as many progenitors as are allowed by the inferred mass range.

The possible progenitors are given in Table 6. The first three columns in this table are the initial mass of the main-sequence progenitor of the white dwarf $\left(M_{0}\right)$ and the mass and radius of the star at the time the evolution is stopped $\left(M_{i}\right.$ and $\left.R_{i}\right)$. The evolution time of the star until this point is given as $t_{\text {evol }}$. The first five possibilities are stars that reach the required core mass while on the AGB, while the last one is peculiar: it reached the required core mass when it had a non-degenerate core. If the progenitor were such a star, the system would have come out of the 
Table 6. Parameters of different white dwarf progenitors at the time the core mass reaches $0.52 M_{\odot}$.

\begin{tabular}{llllllll}
\hline \hline $\begin{array}{l}M_{0}\left(M_{\odot}\right) \\
(1)\end{array}$ & $\begin{array}{l}M_{i}\left(M_{\odot}\right) \\
(2)\end{array}$ & \multicolumn{1}{l}{$\begin{array}{l}R_{i}\left(R_{\odot}\right) \\
(3)\end{array}$} & $\begin{array}{l}t_{\text {evol }}(\mathrm{yr}) \\
(4)\end{array}$ & \multicolumn{1}{c}{$\begin{array}{l}a_{i}\left(R_{\odot}\right) \\
(5)\end{array}$} & \multicolumn{1}{c}{$P_{i}(\mathrm{~d})$} & \multicolumn{1}{l}{$\begin{array}{l}\alpha_{\mathrm{CE}} \lambda \\
(7)\end{array}$} & \multicolumn{1}{l}{$\begin{array}{l}t_{\text {tot }}(\mathrm{yr}) \\
(8)\end{array}$} \\
\hline 1.051 & 0.923 & 231.165 & $6.51 \times 10^{9}$ & 513.052 & 1166.530 & 0.03 & $2.61 \times 10^{10}$ \\
1.191 & 1.085 & 224.726 & $4.95 \times 10^{9}$ & 483.386 & 1007.611 & 0.05 & $2.45 \times 10^{10}$ \\
1.319 & 1.230 & 218.584 & $3.87 \times 10^{9}$ & 459.140 & 890.428 & 0.08 & $2.34 \times 10^{10}$ \\
1.439 & 1.365 & 212.881 & $3.10 \times 10^{9}$ & 438.689 & 799.406 & 0.11 & $2.27 \times 10^{10}$ \\
1.555 & 1.494 & 207.045 & $2.54 \times 10^{9}$ & 419.770 & 722.411 & 0.14 & $2.21 \times 10^{10}$ \\
3.400 & 3.399 & 40.222 & $2.74 \times 10^{8}$ & 71.425 & 35.849 & 5.83 & $1.99 \times 10^{10}$ \\
\hline
\end{tabular}

Notes: Col. (1): initial mass of the main-sequence progenitor; Cols. (2) and (3): mass and radius of the star when it has reached the required core mass. Col. (4): time between the birth of the binary and the start of the CE phase; Cols. (5) and (6): initial orbital separation and initial orbital period; Col. (7): fraction of the orbital period used for ejection of the $\mathrm{CE}$, where $\lambda$ is a numerical value dependent on the structure of the star; Col. (8): time from a main-sequence binary to a CV.

common-envelope phase as a helium-burning star (most likely observable as a subdwarf B star) with a low-mass companion. Only after most helium was burned to carbon and oxygen would the star have turned into a white dwarf.

There are no possible progenitors with initial masses between 1.6-3.4 $M_{\odot}$. For mass $>1.6 M_{\odot}$, the core mass of the giant on the red giant branch (RGB) has not yet grown to the required mass. After the RGB phase, the star contracts again, while the core mass is growing. When the star reaches the AGB phase, the core mass is already larger than the required mass. This results in no possible progenitor star. At the moment of sufficient core mass, the star needs to be larger than any time previously in its evolution, otherwise the star would have started Roche lobe overflow earlier in its evolution when the core mass was not yet massive enough. For stars $>3.4 M_{\odot}$ the core of the giant at the start of the RGB phase is already larger than the required core mass, leaving no possible progenitor for the present-day white dwarf. The progenitor with an initial mass of $3.4 M_{\odot}$ is just on the edge of being a possible progenitor for the present-day white dwarf in DE CVn.

\subsection{Common-envelope phase}

We assume that a possible progenitor fills its Roche lobe at the start of the CE phase. By using the formula for the Roche lobe of Eggleton (1983)

$$
\frac{R_{\mathrm{L}}}{a}=\frac{0.49 q^{\frac{2}{3}}}{0.6 q^{\frac{2}{3}}+\ln \left(1+q^{\frac{1}{3}}\right)}
$$

(with $q=M_{i} / M_{2}$ ), we can calculate the orbital separation $\left(a_{i}\right)$ (and thus the orbital period $P_{i}$ ) at the moment the evolution was stopped. These values are given in Table 6 as well. From the initial orbital period $\left(P_{i}\right.$, Table 6$)$ and the present-day orbital period, we note that a large orbital shrinkage has taken place. Together with the rather extreme initial mass ratios, this implies that DE CVn went through a common-envelope phase during its evolution.

We take the parameters of the giants derived above as the ones at the start of the common-envelope phase. With the use of (de Kool et al. 1987)

$$
\frac{G M_{i}\left(M_{i}-M_{\mathrm{c}}\right)}{R_{i}}=\alpha_{\mathrm{CE}} \lambda\left(\frac{G M_{\mathrm{c}} M_{2}}{2 a_{f}}-\frac{G M_{i} M_{2}}{2 a_{i}}\right),
$$

we calculate $\alpha_{\mathrm{CE}} \lambda$, where $\lambda$ is a numerical value dependent on the structure of the star and $\alpha_{\mathrm{CE}}$ the fraction of the orbital energy that is used for ejection of the common envelope (de Kool 1992), for every possible progenitor. $\alpha_{\mathrm{CE}}$ is usually $0 \leq \alpha_{\mathrm{CE}} \leq 1$, but for double white dwarfs it is found to be $\geq 1$ (Nelemans \& Tout 2005). The $\alpha_{\mathrm{CE}} \lambda$ values for the possible progenitor stars are given in Table 6 as well.

The most massive progenitor has $\alpha_{\mathrm{CE}} \lambda$ of 5.83. Dewi \& Tauris (2000) give $\lambda$ values as a function of the radius of the giant for stars with $M \geq 3 M_{\odot}$. From their table we conclude that for our possible progenitor of mass $3.4 M_{\odot} \lambda$ is around 0.6-0.9, indicating $\alpha_{\mathrm{CE}} \gtrsim 6$, which is very unlikely. Therefore we rule out such a star as the possible progenitor.

The other possible progenitors have $\alpha_{\mathrm{CE}} \lambda<1$, indicating a rather inefficient ejection of the $\mathrm{CE}$ (e.g., a large fraction of the binding energy is not used for ejection of the CE). These values are in agreement with the values found for other white dwarf-red dwarf binaries (e.g., Nelemans \& Tout 2005; Morales-Rueda et al. 2005), and we conclude that the progenitor star of the present-day white dwarf was a main-sequence star with $M \leq 1.6 M_{\odot}$.

\subsection{Time scales}

Now that we can reconstruct the evolution of DE CVn we can determine the corresponding time scales. From the possible progenitors of the white dwarf we find that the time it took before the CE phase started is $2.5-6.5 \times 10^{9}$ years. The temperature of the white dwarf is $8000 \pm 1000 \mathrm{~K}$. From the cooling tracks of Wood (1995) as shown in Fig. 4 of Schreiber \& Gänsicke (2003), we can determine a cooling age $\left(t_{\text {cool }}\right)$ of the white dwarf of $\sim 8 \times 10^{8}$ years. The current age of the system is therefore $3.3-7.3 \times 10^{9}$ years.

Due to angular momentum loss, the system will evolve towards a semi-detached cataclysmic variable (CV) phase. To determine the time that it will take for DE CVn to become a semidetached binary, we first need to know the orbital period at which mass transfer starts $\left(P_{\text {sd }}\right)$. This period follows from the Roche geometry and Kepler's third law:

$P_{\text {sd }}=2 \pi\left(\frac{R_{2}^{3}}{G M_{2}\left(1+\frac{M_{1}}{M_{2}}\right)\left(\frac{R_{\mathrm{L}_{2}}}{a}\right)^{3}}\right)^{0.5}$.

For DE CVn we derive an orbital period at the start of mass transfer of 3 hours.

The orbital period will shrink due to loss of orbital angular momentum. For low-mass stars it is often assumed that the dominant mechanism is (disrupted) magnetic breaking (Verbunt \& Zwaan 1981; King 1988). In short, disrupted magnetic breaking occurs in a close binary when the tides force the secondary star 
to be co-rotating with the binary, while magnetic fields in the secondary force the stellar wind to co-rotate with the secondary star. When this exerts a spin-down torque on the secondary star, it must extract angular momentum from the binary orbit. At a mass of $\sim 0.3 M_{\odot}$, the secondary becomes fully convective and therefore loses its dynamo (or at least changes it) so that magnetic breaking is no longer the dominant source of angular momentum loss.

By assuming that the angular momentum loss is due to disrupted magnetic braking only until the secondary becomes fully convective at a secondary mass of at least $0.3 M_{\odot}$, the time it will take before mass transfer starts is (Schreiber \& Gänsicke 2003):

$t_{\text {sd }}^{2}=\frac{2.63 \times 10^{29} G^{\frac{2}{3}} M_{1}}{(2 \pi)^{\frac{10}{3}}\left(M_{1}+M_{2}\right)^{\frac{1}{3}}} R_{2}^{-2} \times\left(P_{\text {orb }}^{\frac{10}{3}}-P_{\text {sd }}^{\frac{10}{3}}\right)$,

where $M_{1}$ and $M_{2}$ are in $M_{\odot}$ and $R_{2}$ in $R_{\odot}$. This corresponds to a time of $1.9 \times 10^{10}$ years before DE CVn becomes a CV, which is just longer than the Hubble time. Systems such as DE CVn will not contribute to the current sample of CVs, unless the loss of angular momentum in the current detached white dwarf-red dwarf phase is much higher than that given by magnetic braking alone (see, e.g., Brinkworth et al. 2006, where an angular momentum loss mechanism $\sim 100$ times greater in strength than the currently accepted value seems to be required to explain the rate of period decrease in NN Ser).

Acknowledgements. We thank Pierre Bergeron for making his cool white dwarf models available to us. EvdB, LMR, and PJG are supported by NWOVIDI grant 639.042.201 to P.J. Groot. GN is supported by NWO-VENI grant 638.041.405 to G. Nelemans. JRT thanks the US National Science Foundation for support through grants AST-9987334 and AST-0307413. Tim Miller and Maddie Reed took some of the MDM photometric observations, and Bill Fenton took some of the MDM spectra. TRM was supported by a PPARC Senior Fellowship during the course of this work. ULTRACAM is supported by PPARC grants PP/D002370/1 and PPA/G/S/2003/00058. The William Herschel Telescope is part of the Isaac Newton Group of Telescopes operated on the island of La Palma by the Instítuto de Astrofísica de Canarias on behalf of the British PPARC and the Dutch NWO. We acknowledge the use of the $0.5 \mathrm{~m}$ telescope of the Cimenhage Observatory and the $1.8 \mathrm{~m}$ telescope of the Dominion Astrophysical Observatory located in Victoria, Canada. We acknowledge the use of the $1.3 \mathrm{~m}$ telescope and the $2.4 \mathrm{~m}$ Hiltner Telescope of the MichiganDartmouth-MIT Observatory in Arizona and the $2 \mathrm{~m}$ Alfred-Jensch-Teleskop at the Thüringer Landessternwarte.

\section{References}

Bergeron, P., Wesemael, F., \& Fontaine, G. 1991, ApJ, 367, 253 Bergeron, P., Saumon, D., \& Wesemael, F. 1995, ApJ, 443, 764

Brinkworth, C. S., Marsh, T. R., Dhillon, V. S., \& Knigge, C. 2006, MNRAS, 365,287

Bruch, A. 1999, AJ, 117, 3031

de Jager, C., Heise, J., Avgoloupis, S., et al. 1986, A\&A, 156, 95

de Jager, C., Heise, J., van Genderen, A. M., et al. 1989, A\&A, 211, 157 de Kool, M. 1992, A\&A, 261, 188

de Kool, M., van den Heuvel, E. P. J., \& Pylyser, E. 1987, A\&A, 183, 47

Dehnen, W., \& Binney, J. J. 1998, MNRAS, 298, 387

Dewi, J. D. M., \& Tauris, T. M. 2000, A\&A, 360, 1043

Dhillon, V., \& Marsh, T. 2001, New Astron. Rev., 45, 91

Eggleton, P. P. 1983, ApJ, 268, 368

Fuhrmann, K. 2004, Astronomische Nachrichten, 325, 3

Green, R. F., Schmidt, M., \& Liebert, J. 1986, ApJS, 61, 305

Haefner, R. 1989, A\&A, 213, L15

Holmes, S., \& Samus, N. 2001, J. Am. Association of Variable Star Observers (JAAVSO), 29, 148

Hurley, J. R., Pols, O. R., \& Tout, C. A. 2000, MNRAS, 315, 543

Johnson, D. R. H., \& Soderblom, D. R. 1987, AJ, 93, 864

King, A. R. 1988, QJRAS, 29, 1

Kirkpatrick, J. D., Henry, T. J., \& McCarthy, D. W. 1991, ApJS, 77, 417

Marsh, T. R. 2000, New Astron. Rev. , 44, 119

Maxted, P. F. L., Marsh, T. R., Morales-Rueda, L., et al. 2004, MNRAS, 355, 1143

Monet, D. G., Levine, S. E., Canzian, B., et al. 2003, AJ, 125, 984

Morales-Rueda, L., Marsh, T. R., Maxted, P. F. L., et al. 2005, MNRAS, 359, 648

Nelemans, G., \& Tout, C. A. 2005, MNRAS, 356, 753

O’Donoghue, D., Koen, C., Kilkenny, D., et al. 2003, MNRAS, 345, 506

Paczyński, B. 1976, in Structure and Evolution of Close Binary Systems, IAU Symp., 73, 75

Panei, J. A., Althaus, L. G., \& Benvenuto, O. G. 2000, A\&A, 353, 970

Pickles, A. J. 1998, PASP, 110, 863

Reid, I. N., Hawley, S. L., \& Gizis, J. E. 1995, AJ, 110, 1838

Robb, R. M., \& Greimel, R. 1997, Information Bulletin on Variable Stars, 4486, 1

Schreiber, M. R., \& Gänsicke, B. T. 2003, A\&A, 406, 305

Smith, J. A., Tucker, D. L., Kent, S., et al. 2002, AJ, 123, 2121

Taam, R. E., \& Sandquist, E. L. 2000, ARA\&A, 38, 113

Tas, G., Sipahi, E., Dal, H. A., et al. 2004, Information Bulletin on Variable Stars, 5548,1

Verbunt, F., \& Rappaport, S. 1988, ApJ, 332, 193

Verbunt, F., \& Zwaan, C. 1981, A\&A, 100, L7

Voges, W., Aschenbach, B., Boller, T., et al. 1999, A\&A, 349, 389

Wesemael, F., Greenstein, J. L., Liebert, J., et al. 1993, PASP, 105, 761

Wood, M. A. 1995, in LNP Vol. 443, White Dwarfs, ed. D. Koester \& K. Werner, 41 